\title{
Inverse Additive Number Theory. XI. Long arithmetic progressions in sets with small sumsets
}

by

\author{
Gregory A. Freiman (Tel Aviv)
}

This paper continues the series of papers on Inverse Additive Number Theory published in 1955-1964 (see references [84]-[92], [98] in [2]).

Throughout the paper, we work with the set $A \subset \mathbb{Z}$ of cardinality $|A|=$ $k \geq 3$. We assume that

$$
A=\left\{a_{0}=0<a_{1}<\cdots<a_{k-1}\right\}
$$

and that the greatest common divisor of the numbers from $A$ is 1 . Let $T$ denote the cardinality of the set $2 A=A+A$ of all pairwise sums $a+b$ of numbers from $A$. Notice that $T \geq 2 k-1$.

In [1] (see also the textbook [3, p. 204]), we proved the following result.

Theorem 1. For $0 \leq b<k-2$ and $T=2 k-1+b$, the set $A$ is contained in

$$
L=\{0,1,2, \ldots, k+b-1\} .
$$

Let us give several examples of such sets for the maximal value of $b=$ $k-3, T=3 k-4$ and $k=8$ :

$$
\begin{aligned}
& A=\{0,2,4,6,8,10,11,12\}, \\
& A=\{0,2,4,6,7,8,10,12\}, \\
& A=\{0,6,7,8,9,10,11,12\} .
\end{aligned}
$$

The fact that a set $A$ with a small doubling (small $T$ ) may be included in a short interval reflects only part of the whole picture.

In order to formulate the main result of the paper we define several new notions.

Let $e$ denote the maximal $a \in\left[0, a_{k-1}\right]$ with $a \notin 2 A$; if the interval $\left[0, a_{k-1}\right]$ is included in $2 A$, then we put $e=-1$. 
Let $c$ denote the minimal $a \in\left[0, a_{k-1}\right]$ with $a+a_{k-1} \notin 2 A$; if the interval $\left[a_{k-1}, 2 a_{k-1}\right]$ is included in $2 A$, then we put $c=a_{k-1}+1$.

In Lemma 6 we show that one always has

$$
e<c \text {. }
$$

We also need the following definition: the set $A$ is called stable if

$$
2 A \cap\left[0, a_{k-1}\right]=A .
$$

Examples of stable sets: $\{0,6\},\{0,2,4,6\},\{0,3,4,5\}$.

Define

$$
B=A \cup\left(a_{k-1}+A\right) .
$$

We have $B \subset M_{1}$, where $M_{1}=\left[0,2 a_{k-1}\right]$.

Let $C$ be a set of integers. If $x \in[\min C, \max C] \backslash C$, then we say that $x$ is a hole in $C$. For example, in (2) we have $A \subseteq M=[0,12]$ and the set of holes is $\{1,3,5,7,9\}$. Note that if $a$ is a hole in $A$, then $\left(a, a+a_{k-1}\right)$ is a pair of holes in $B$; in what follows we will use only such pairs, i.e. $a \notin A$.

We can now formulate the main result of this paper:

TheOREM 2. In the setting of Theorem 1, we have

$$
J=\left[e+1, c+a_{k-1}-1\right] \subset 2 A,
$$

and

$$
|J| \geq 2 k-1+2 d,
$$

where $d$ is the number of holes in $A$ in the open interval $(e, c)$.

The most interesting result is when we assume that the interval containing $A$ has the maximal length for a given $T$. The following assertion is a consequence of Theorem 2 :

Corollary 1. If $T=|A+A|=2 k-1+b$ where $0 \leq b<k-2$ and if

$$
a_{k-1}=k-1+b,
$$

then

(a) $A_{1}=A \cap[0, e+1]$ is stable, i.e. $A \cap[0, e]=2 A \cap[0, e]$,

(b) $A_{2}=a_{k-1}-\left(\left[c-1, a_{k-1}\right] \cap A\right)$ is stable,

(c) $J=\left[e+1, c+a_{k-1}-1\right] \subset 2 A$,

(d) $I=[e+1, c-1] \subset A$.

We see that in this case the set $A$ may be partitioned into three parts,

$$
A=A_{1} \cup I \cup\left(a_{k-1}-A_{2}\right),
$$

where $A_{1}$ and $A_{2}$ are stable, and $I$ is an interval, and the set $2 A$ may be partitioned into three parts,

$$
2 A=A_{1} \cup J \cup\left(2 a_{k-1}-A_{2}\right),
$$

where $J$ is an interval. 
We define the length of an interval of integers (or of an arithmetic progression) to be the number of his elements. So, the length of $L$ in (1) is $|L|=|[0, k+b-1]|=k+b$.

We denote by $M$ or $M(A)$ the minimal interval containing $A$. From Theorem 1 it follows that

$$
a_{k-1}=k-1+b^{\prime}
$$

where

$$
b^{\prime} \leq b .
$$

Thus, the length of $M=\left[0, a_{k-1}\right]$ is equal to $k+b^{\prime}$. We will now estimate $b^{\prime}$ from below. From $A \subset\left[0, k-1+b^{\prime}\right]$ it follows that

$$
2 A \subseteq 2\left[0, k-1+b^{\prime}\right]=\left[0,2 k-2+2 b^{\prime}\right]
$$

and

$$
|2 A| \leq\left|\left[0,2 k-2+2 b^{\prime}\right]\right|=2 k-1+2 b^{\prime} .
$$

Thus, from $|2 A|=T=2 k-1+b$, we get

$$
b^{\prime} \geq b / 2 .
$$

From $A \subset M=\left[0, a_{k-1}\right]$ and (5), we see that the number of holes in $A$ is equal to $b^{\prime}$. We have

$$
B \subset M_{1}=\left[0,2 a_{k-1}\right]
$$

From $|B|=2 k-1$ and

$$
\left|M_{1}\right|=2 a_{k-1}+1=2\left(k-1+b^{\prime}\right)+1=2 k-1+2 b^{\prime},
$$

it follows that the number of holes in $B$ is equal to $2 b^{\prime}$.

The following Lemmas 2-6 will be used in the proof of Theorem 2 .

Lemma 2. For each pair $\left(a, a+a_{k-1}\right)$ of holes in $B$ we have either

$$
a \in 2 A,
$$

or

$$
a+a_{k-1} \in 2 A .
$$

Proof. Let us look at $A$ as a set of residues modulo $a_{k-1}$. Our modulus, $a_{k-1}$, has $k+b^{\prime}-1 \leq k+b-1 \leq 2 k-4$ residues, and the sets $A\left(\bmod a_{k-1}\right)$ and $a-A\left(\bmod a_{k-1}\right)$ contain $k-1$ residues each, because the numbers 0 and $a_{k-1}$ are congruent modulo $a_{k-1}$. Thus, the sets of residues $A$ and $a-A$ have a non-zero intersection, and therefore

$$
a \in 2 A\left(\bmod a_{k-1}\right) .
$$

But in the set of integers the residue $a$ is represented by $a$ or by $a+a_{k-1}$. If neither of these numbers belongs to $2 A$ then this contradicts (12). Therefore we have (10) or (11). 
For the pair $\left(a, a+a_{k-1}\right)$ of Lemma 2 , one of the numbers of the pair belongs to $2 A$. And the other one?

DEFINITION. If both numbers in the pair $\left(a, a+a_{k-1}\right)$ belong to $2 A$, i.e. (10) and (11) are valid, we call the pair unstable. This pair is called stable if one of the numbers of the pair does not belong to $2 A$, and this number will be called a stable hole. If

$$
a \notin 2 A,
$$

the pair will be called left; if

$$
a+a_{k-1} \notin 2 A,
$$

the pair will be called right.

The number and location of pairs of different types depends to a large extent, as we will see, on the structure of both $2 A$ and $A$.

The number

$$
b^{\prime}=a_{k-1}-k+1
$$

represents the number of holes in $A$ and at the same time the number of pairs of holes $\left(a, a+a_{k-1}\right)$ in $B$.

Lemma 3. In $B$, there are $2 b^{\prime}-b$ stable pairs of holes and $b-b^{\prime}$ unstable pairs of holes.

Proof. The number of holes in $B$ is equal to $2 b^{\prime}$. To get all $2 k-1+b$ numbers of $2 A$ we have to add, to the $2 k-1$ numbers of $B, b$ more numbers, which are holes in $B$, so that the number of stable holes is equal to $2 b^{\prime}-b$, and the same is the number of stable pairs (one stable hole in a stable pair). The whole number of pairs of holes in $B$ is equal to $b^{\prime}$. The number of unstable pairs is equal to

$$
b^{\prime}-\left(2 b^{\prime}-b\right)=b-b^{\prime} .
$$

In the next two lemmas, which are immediate consequences of the pigeonhole principle, we begin to explain why the holes in $A$ under the conditions of Theorem 1 are concentrated in the neighborhoods of the endpoints of $M=\left[0, a_{k-1}\right]$.

Lemma 4. If $a \notin 2 A$, then the number of holes of $A$ which belong to the interval $[0, a]$ is greater than or equal to $[a / 2+1]$.

Lemma 5. The number of holes in $A$ which belong to an interval $I=$ $\left[a, a_{k-1}\right]$ when $a+a_{k-1}$ is a right stable hole is greater than or equal to $\left[\left(a_{k-1}-a\right) / 2+1\right]$.

We are now ready to prove that the numbers in the set of left stable holes are smaller than the numbers in the set of right stable holes; the set of numbers between these two sets in $A$ contains only holes which are unstable, and this ensures the existence of a long interval in $2 A$. 
LEMma 6. We have

$$
e<c \text {. }
$$

Proof. We know that $e$ is stable in a left stable pair and so $e \notin 2 A$, and from the fact that $c$ is stable in a right stable pair $\left(c, c+a_{k-1}\right)$, we get $c+a_{k-1} \notin 2 A$.

If $e=c$, then the pair $\left(e, e+a_{k-1}\right)$ would have neither element in $2 A$, in contradiction to Lemma 1.

Suppose now, contrary to the conclusion, that $e>c$.

The number of holes in $A$ is equal to $b^{\prime}$. We will estimate this number from below, using estimates of the values $e$ and $c$.

Now we build a finite sequence of pairs of numbers

$$
\left(c_{1}, e_{1}\right), \ldots,\left(c_{i}, e_{i}\right)
$$

in the following manner.

Define $c_{1}=c, e_{1}=e$. Suppose that the pair $\left(c_{j}, e_{j}\right)$ is already built, where $c_{j}$ is a stable point from a right stable pair and $e_{j}$ is a stable point from a left stable pair. There are the following possibilities:

(i) There exists a left stable pair $\left(a, a+a_{k-1}\right)$ such that $c_{j}<a<e_{j}$.

(ii) Case (i) is not valid but there exists a right stable pair $\left(a, a+a_{k-1}\right)$ such that $c_{j}<a<e_{j}$.

(iii) Cases (i) and (ii) are not valid.

In case (i) put $c_{j+1}=c_{j}, e_{j+1}=a$; if (ii) is true put $c_{j+1}=a, e_{j+1}=e_{j}$; if (iii) is true put $j=i$, and the sequence is built. Let us mention that the sequence (15) was built in such a way that

$$
c_{j}<e_{j}, \quad j=1, \ldots, i, \quad\left[c_{1}, e_{1}\right] \supset \cdots \supset\left[c_{i}, e_{i}\right] .
$$

Denote by $x$ the number of holes in $A$ which belong to the interval $\left(c_{i}, e_{i}\right)$. All these holes, because of the manner in which we built them, are unstable, and we have, because of Lemma 4, an estimate

$$
x \leq b-b^{\prime} .
$$

We clearly have

$$
x \leq e_{i}-c_{i}-1 .
$$

The holes in $A$ which are in the interval $\left[c_{i}, e_{i}\right]$ are perhaps counted twice when we estimate the number of holes in $A$ belonging to $\left[1, e_{i}\right]$ with the help of Lemma 4 and when we estimate the number of holes in $A$ belonging to $\left[c_{i}, a_{k-1}\right]$ with the help of Lemma 5 . Putting all what has been said together we obtain the inequality

$$
b^{\prime} \geq\left(e_{i}+1\right) / 2+\left(a_{k-1}-c_{i}+1\right) / 2-x-2 .
$$


In view of (5), we get

$$
b^{\prime} \geq\left(k+b^{\prime}\right) / 2+\left(e_{i}-c_{i}\right) / 2-3 / 2-x
$$

and therefore

$$
b^{\prime} \geq k+e_{i}-c_{i}-3-2 x .
$$

Using (17) we get

$$
b^{\prime} \geq k-2-x+e_{i}-c_{i}-1-x \geq k-2-x .
$$

Because of (16) we have

$$
0 \geq k-b+\left(b-b^{\prime}\right)-x-2 \geq k-b-2 \geq k-(k-3)-2=1,
$$

a contradiction.

Proof of Theorem 2. We will now use Lemmas 4-6 to estimate the length of the interval contained in $2 A$. We will show that

$$
J=\left[e+1, c+a_{k-1}-1\right] \subset 2 A
$$

and

$$
|J| \geq 2 k-1+2 d,
$$

where $d$ is the number of holes in $A$ in the interval $(e, c)$.

We first prove that (18) is valid. Let $f \in J$. If $f \in B$ then $f \in 2 A$, because $B \subseteq 2 A$. If $f \notin B$, then $f$ is one of the numbers of the pair $\left(a, a+a_{k-1}\right)$. If this pair is unstable, then both numbers in it belong to $2 A$ and so $f \in 2 A$. If this pair is left stable, then $a \notin 2 A$ and $a \leq e$. Thus, $f=a+a_{k-1} \in 2 A$. If this pair is right stable, then $a+a_{k-1} \notin 2 A$ and $a \geq c$. Thus, $f=a \in 2 A$.

We now prove the estimate (19). From (18) and (5) we get

$$
|J|=c+a_{k-1}-1-e=k-2+b^{\prime}+c-e .
$$

We now estimate $c-e$ from below. For this we will estimate the number $P$ of holes in $A$ which are less than $e$ or larger than $c$. For the number $P_{1}$ of holes which are less than $e$ we have, according to Lemma 4,

$$
P_{1} \geq(e+1) / 2,
$$

and for the number $P_{2}$ of holes which are greater than $c$ we have, according to Lemma 5,

$$
P_{2} \geq\left(a_{k-1}-c+1\right) / 2 \text {. }
$$

The sets $P_{1}$ and $P_{2}$ have an empty intersection, in view of Lemma 6 , and thus, in view of (21) and (22),

$$
P \geq P_{1}+P_{2} \geq(e+1) / 2+\left(k-1+b^{\prime}-c+1\right) / 2 .
$$

We will get an estimate of $P$ from above by taking the number of all pairs $b^{\prime}$ minus the number $d$ of those $a$ which are holes in $A$ in the interval $(e, c)$. 
Thus,

$$
b^{\prime}-d \geq\left(e+k+b^{\prime}-c+1\right) / 2
$$

and

$$
c-e \geq k+b^{\prime}+1-2\left(b^{\prime}-d\right)=k+2 d+1-b^{\prime} .
$$

Because of (24) we get from (20)

$$
|J| \geq k-2+b^{\prime}+k+2 d+1-b^{\prime}=2 k+2 d-1 .
$$

Proof of Corollary 1. We have $b^{\prime}=b$. Thus the set of unstable pairs is empty, every point of the interval $\left[e+1, c+a_{k-1}-1\right]$ belongs to $2 A$.

The elements of $[0, e+1]$ which are holes in $A$ may belong only to a left stable pair, and so the set

$$
A_{1}=[0, e+1] \cap A
$$

is stable. A similar reasoning may be applied to $A_{2}$.

EXAMPLE.

$$
A=\{0,2,4,6,7,8,9,10,14\} .
$$

We have here $e=5, c=11$, the set $A_{1}=\{0,2,4,6\}$ is stable, the set $A_{2}=\{0,4\}$ is stable, $J=[6,24]$ and $I=\{6,7,8,9,10\}$.

\section{References}

[1] G. A. Freiman, The addition of finite sets I, Izv. Vyssh. Uchebn. Zaved. Mat. 6 (1959), no. 13, 202-213 (in Russian).

[2] -, Structure theory of set addition, Astérisque 258 (1999), 1-30.

[3] M. B. Nathanson, Additive Number Theory. Inverse Problems and the Geometry of Sumsets, Grad. Texts in Math. 165, Springer, New York, 1996.

School of Mathematical Sciences

Tel Aviv University

Tel Aviv 69978, Israel

E-mail: grisha@post.tau.ac.il

Received on 3.2.2008

and in revised form on 27.1.2009 